Recibido 05 de Enero, 2019 - Aceptado 05 de Febrero, 2019

\title{
Informe herpetología para Ucayali, Perú - puntos base
}

\section{Herpetology report for Ucayali, Peru - points base}

Brian Crnobrna ${ }^{1}$

\section{RESUMEN}

Solo hay dos estudios formales enfocados en anfibios y reptiles del Valle Alto-Ucayali. Estos estudios tienen implicacias fuera del estudio de herpetología, tal como indicadores ambientales, áreas protegidas, y el uso sostenible de recursos naturales. A la fecha, el uso de búsquedas generales domina la metodología de herpetología en Ucayali, pero transectos también han sido ingresados. La variación de bosques, entre partes bajíos y el terreno firme, explica gran parte de las diferencias en diversidad entre sitios, especialmente en los grupos de ranas venenosas (familia Dendrobatidae) y el género Osteocephalus. Un estimado de las especies registradas en el Valle Alto-Ucayali llega a 114 especies, indicando que una gran diversidad queda para descubrir.

Palabra claves: Anfibios, reptiles, herpetología, Ucayali, monitoreo

\section{ABSTRACT}

There are only two formal studies focused on amphibians and reptiles of the AltoUcayali Valley. These studies have implications outside of the just study of herpetology, such as environmental indicators, protected areas, and the sustainable use of natural resources. To date, the use of general searches dominates the methods of herpetology in Ucayali, but transects have also been entered. The variation in forests, between lowlands and terra firma, explains in large part the differences in diversity between sites, especially in the poison dart frog group (family Dendrobatidae) and the genus Osteocephalus. An estimate of all the species registered in the Alto-Ucayali Valley reaches only 114 species, indicating that much diversity remains to be discovered.

Keyword: Amphibians, reptiles, herpetology, Ucayali, monitoring

1. Coordinador de Herpetofauna, Asociación Fauna Forever, Secretario, Instituto Amazónico para la Innovación y Desarrollo Sostenible, Escolar de Desarrollo Global, Universidad Nacional de Ucayali

Email: tripanurgus@gmail.com 


\section{INTRODUCCIÒN}

La "línea base y protocolo de monitoreo ambiental en al zona de influencia Sierra del Divisor" era el primer paso en destapar el estado de anfibios y reptiles en la cuenca del Río Alto-Ucayali. Este estudio fue desarrollado en dos sitios de la cuenca Abujao. Las tareas estaban enfocados en la temporada seca del año 2015 (1), de origen la Facultad de Ingeniera Forestal y Ambiental de la Universidad Nacional de Ucayali (UNU).

El diseño para estudiar la zona Abujao era una cooperación entre la UNU y las comunidades nativas para encontrar cuencas potenciales de conflictos entre pobladores e instituciones. También estaban involucrados estudiantes de la UNU para terminar tales prácticas.

El segundo paso en estudiar herpetología en el región Alto-Ucayali tuvo como antecedente un acta de registros publicado por el Museo Nacional en Praga, República Checa. Las únicas actividades de este estudio fue dos expediciones de colección en la zona del Área de Conservación Regional (ACR) Imiría (2), desarrollado por el equipo técnico del Museo, dedicándose el periodo de cinco años en procesar los datos de recolección, y publicó en el año 2017.

Estos dos estudios integran la vista completa de herpetología en la cuenca Alto-Ucayali, como los estudios de Sierra del Divisor (3), Matses (4), Provincia Purus (5), y Cordillera El Sira se ubicaron afuera de la región. Además, se ubican fuera de la influencia del sistema de humedales adyacente al Río Alto-Ucayali, sumamente contenido en el Dpto. Ucayali y las 12 vueltas (sinusoidal por el tramo fluvial) del Río Ucayali más arriba en Dpto. Loreto $(6,7)$.

Los objetivos de estos dos estudios presentan en resumen lo siguiente:

a. El monitoreo de poblaciones de anfibios en Ucayali ocurre sobre un área de bosque húmedo con alto nivel de biodiversidad (8). Tal bosque se encuentra con varios amenazas a su ecología y servicios ambientales, de los cuales también afectan poblaciones de anfibios. Perturbaciones poblacionales de anfibios, que implica un indicador de perturbación ambiental, se puede servir como alerta temprana a los que desarrollan problemas mas graves en el futuro (9).

b. Desarrollando listas de especies herpetológicas se entrega fácil con las metas de las áreas protegidas en la región Ucayali (10). El tipo de evidencia generado por monitoreo de herpetofauna explica factores vinculados a la eficacia de un área protegida en la composición de sus recursos naturales $(11,12)$. Como algunos áreas han sido establecidas en beneficio de proteger especies en amenaza, siendo necesario documentar su biodiversidad.

c. La fauna silvestre también forma parte de los recursos naturales para el uso en subsistencia y comercio de las comunidades locales, conocimiento fundamental de fauna silvestre, que puede reducir probabilidades de conflictos (8).

d. Continuar monitoreo de anfibios y reptiles con la finalidad de crear oportunidades para cooperación institucional, tales como la UAP, la UNU, el Gobierno Regional de Ucayali, SERNANP, y otros ONG involucrados en conservación. De esta manera se ayudara en compartir facilidades de laboratorios, colecciones, y equipos modernos de investigación, para avanzar las metas del estudio.

e. Otro factor a tomar en cuenta es la contribución de herpetofauna en el Ecoturismo. Mientras las actividades de tours con especialidad en herpetología son muy raros en el Perú, reptiles y anfibios siempre tienen papel importante en los tours exitosos (13). La exploración de esta opción en Ucayali es muy recomendable.

\section{MÈTODOS Y MATERIALES}

Los estudios dedicados a los sitios en Ucayali, para el uso de transectos, puede involucrarse con 
un comparación con otros estudios de la Amazonía Peruana con transectos de 50, 100, y 1000 $\mathrm{m}(11,12)$. La restricción de área usando $2 \mathrm{~m}$ en ambos lados, una estrategia a facilitar la metodología, representa un compromiso a las observaciones que se puede aumentar en los 5, 10, o 15 metros de ancho según las muestras-posiblemente empleando un muestreo de distancia diseñado para usar funciones de detección.

En las expediciones con fines de recolección de muestras solo emplean búsquedas generales de tiempo restringida. De esta manera se puede cuantificar normalmente para alcanzar un tamaño de muestra mínimo en varios sitios.

En Abujao hicieron algunos pruebas de muestras auditivos. Este tipo de método es importante para avanzar la descripción de biodiversidad sobre anfibios y otro grupos. Mas que todo se permite la distinción de especies mediante cantos (sonidos producido por ranas machos), a diferencia documentado en el campo de lo obtenido por alguna publicación. Este procedimiento engloba el incremento de cifras de poblaciones según especies.

La colección de muestras herpetológicas implica el proceso de fijación y catálogo en alcohol ejemplares de cada especies encontradas. El proceso de preservación de muestras podrá habilitar mejor exactitud en identificación, estudios genéticos, y descubrimiento de nuevas especies (14).

\section{Variables de los sitios}

Para ampliar la detección de la diversidad de herpetofauna, el diseño espacial que incluye diferentes tipos de bosque es muy importante. En Abujao, el estudio tuvo la finalidad de monitorear un bosque de terraza alta, bosque de terraza baja, bosque ribereño, y terreno firme. En el ACR Imiría, no se encuentra la diversidad de tipos de bosques elaborados a través de imágenes satélites (15-17). El estudio de Moravec (2) asigna muestras pertinentes a solo un tipo: El bosque húmedo siempreverde del suroeste Amazonía. Al mismo tiempo llamaron la atención el contraste de bosques inundados mas cerca de la Laguna Imiría. Estos humedales, y su rol en poblaciones de anfibios, solo aplican como variable en los sitos vinculados al ACR Imiría.

\section{RESULTADOS}

A la fecha, hay 114 especies de anfibios y reptiles identificados en la cuenca Alto-Ucayali. En esta lista no aparece alguna especie categorizada en amenaza por la lista roja del IUCN; aunque el caimán negro (Melanosuchus niger) se encuentra en la lista de CITES como especies prohibida para comercializar.

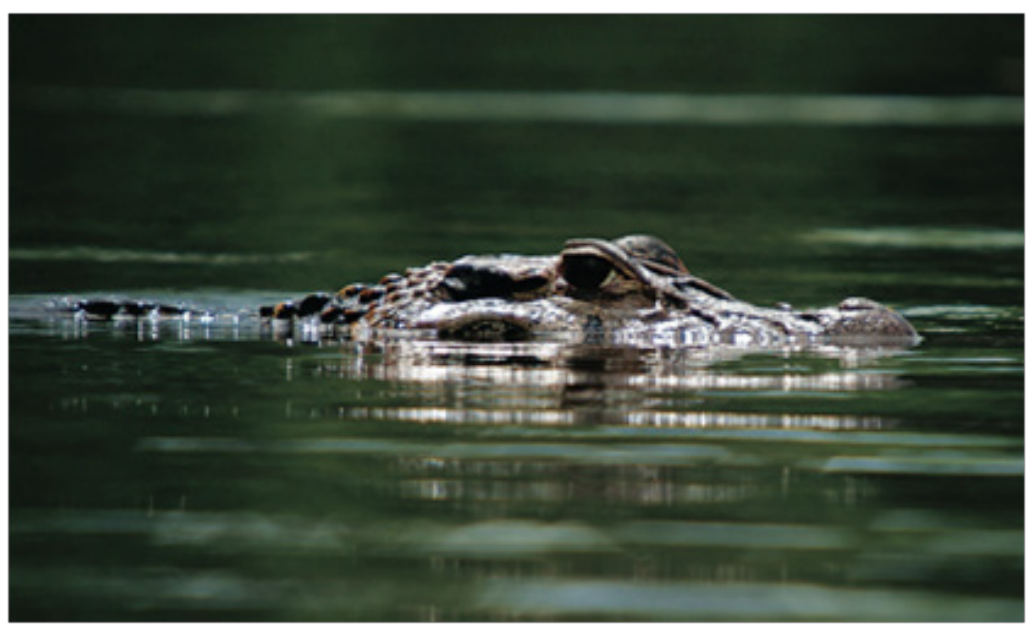

Figura No 1. Caiman negro (Melanosuchus niger)

(foto por Juan Carlos Huayllapuma) 


\section{Sitios Abujao:}

Se ubica la Comunidad Nativa Santa Rosa de Tamaya Tipishca en las orillas del dicho cuerpo del agua, que esta afluente de los ríos Abujao y Tamaya. Se encuentra regularmente las zonas de bosque de inundación estacional de las llanuras meandricas o "tahuampas". Este tipos de bosque normalmente es bajo en diversidad, pero los poblaciones de anfibios están mucho más productivas en cantidades $(18,19)$.

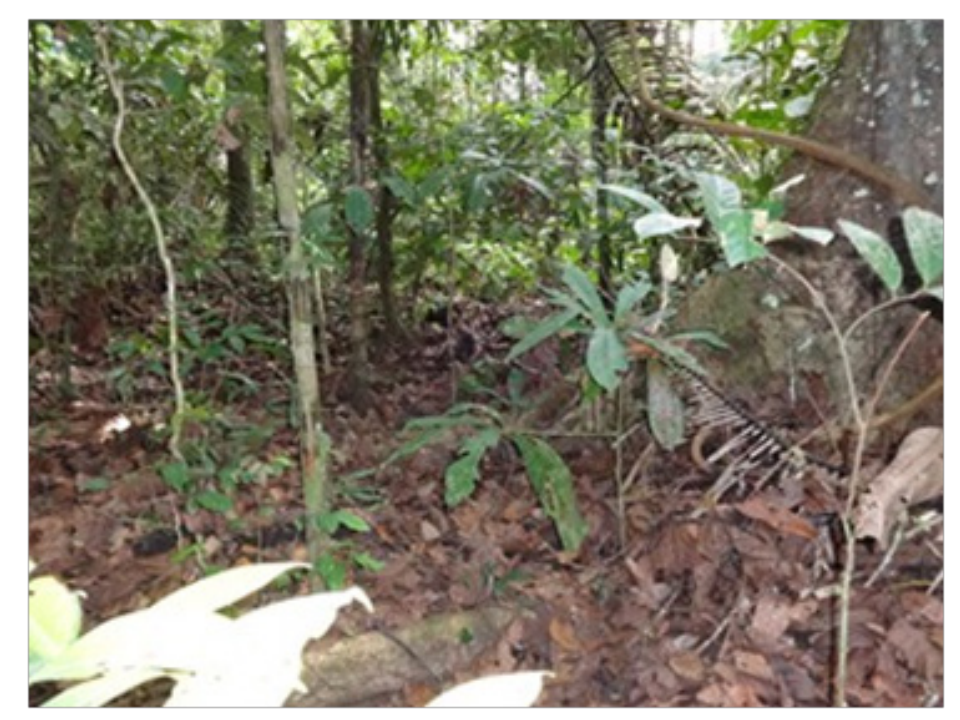

Figura No 2. Bosque de San Mateo

Cerca de a otra comunidad nativa incluido en el estudio, de nombre San Mateo, se encuentra otros tipos de bosque más alto y seco. El bosque de terraza alta o terreno firme, un ambiente con poco agua disponible para los anfibios, esta presión en encontrar las hábitats de cría, que son muy pequeñas. Esto resulta un factor de altos niveles de diversidad $(18,20)$. Debido a esta tendencia San Mateo cuenta con la presencia de tres especies de ranas venosas (1) —especies muy importante que compone todo la diversidad biológico de Ucayali.
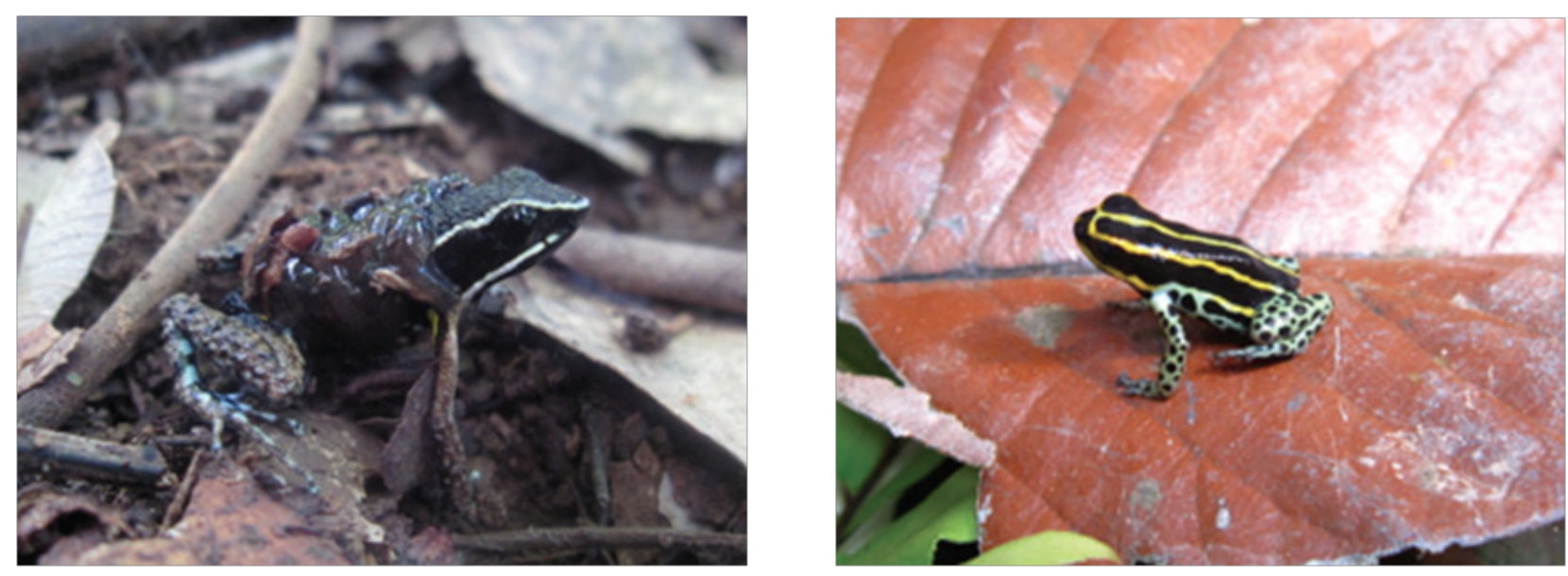

Figura No 3. Ranas venenosas, un grupo poco conocido en Ucayali - los machos llevan sus renacuajos en la espalda, Ameerega hahneli y Ranitomeya biolat, (origen Tambopata, Madre De Dios, Perú) 
Lista de especies:

Herpetofauna (reptiles y anfibios)Abujao basin(Santa Rosa de Tamaya Tipishca y San Mateo)

71 species

\section{Ranas}

\section{Bufonidae}

La familia de los sapos

Rhinella marina

Rhinella margaritifera

\section{Aromobatidae}

La familia de las ranas cohete

Allobates femoralis

\section{Dendrobatidae}

La familia de las ranas venenosas

Ameerega aff. ignipedis

Ameerega trivittata

Ranitomeya sp.

\section{Craugastoridae}

La famiilia de las ranas landron de hoja rasca

Oreobates quixensis

Pristimantis achuar

Pristimantis croceonguinus

Pristimantis ockendeni

Pristimantis peruvianus

Pristimantis sp. 1

Pristimantis sp. 2

Pristimantis sp. 3

\section{Hylidae}

La familia de las ranas arbicolas

Dendropsophus parviceps

Dendropsophus sarayacuensis

Boana boans

Boana cf. maculateralis

Boana geografica

Osteocephalus buckleyi

Osteocephalus aff. yasuni

Osteocephalus planiceps

Osteocephalus taurinus

Phyllomedusa vaillanti

Scinax pedromedinae

Scarthyla goinorum

Sphaenorynchus dorisae

\section{Leptodactylidae}

La familia de las ranas de lluvia

Edalorhina perezi

Adenomera andreae

Leptodactylus bolivianus

Leptodactylus discodactylus

Leptodactylus leptodactyloides

Leptodactylus pentadactylus ("hualo")

Lithodytes lineatus

\section{Microhylidae}

La familia de las ranas de boca estrecho

Hamptophryne boliviana

\section{Caudata}

Salamandras

Bolitoglossa altamazonica

\section{Largartijas}

Dactyloidae

Anolis fuscoauratus

Anolis ortonii

\section{Teiidae}

Dracaena guianensis

Kentropyx altamazonica

Kentropyx pelviceps

\section{Hoplocercidae}

dragon de bosque

Enyaloides palpebralis

\section{Gekkota}

Gekkos

Gonatodes humeralis

Pseudogonatodes

\section{Gymnophthalmidae}

Lagartijas enanas

Cercosaura argulus

Iphisa elegans

Leposoma parietale 


\section{Scincidae}

Copeoglossum nigropunctatum

\section{Tropiduridae}

The family of the tree runners

Plica plica

Plica umbra

\section{Serpientes}

\section{Boidae}

La familia de las Boas

Corallus hortulanus

Corallus batesi

\section{Colubridae}

La familia de las serpientes inofensivas ("afanignas")

Chironius mutliventris

Chironius fuscus

Dendrophidion dendrophis

Drymoluber dichrous

Phrynonax poecilonotus

Sitios acrimiría:

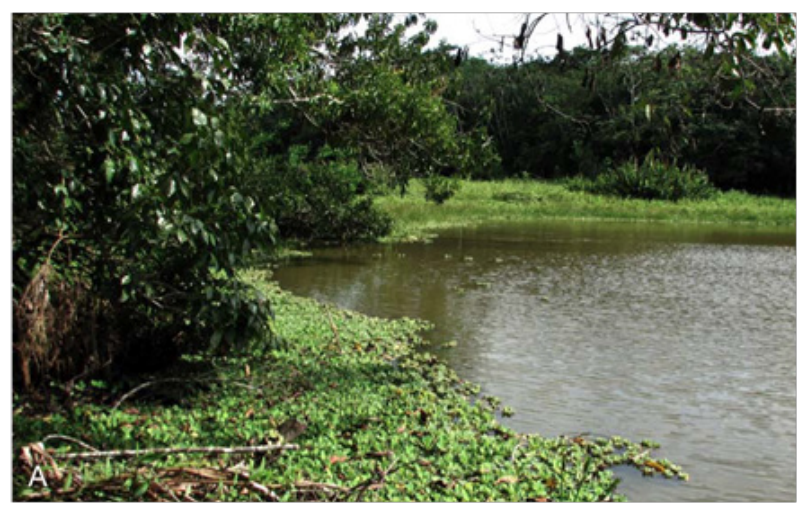

\section{Dipsadidae}

La familia de las culebras

Imantodes cenhcoa

Dipsas catesbyi

Dipsas indica

\section{Sub-familia Xenodontinae}

culebras de colmillos traseros ("mashacuy")

Drepanoides anomolus

Oxyrhopus melanogenys

Oxyrhopus formosus

Pseudoboa coronata

\section{Elapidae}

La familia de las Nakka Nakka

Micrurus annelatus

\section{Viperidae}

La familia de las viboras

Bothrops atrox (“jergon”)

\section{Tortugas}

Podocnemis unifilis ("taricaya")

Chelonoidis denticulate ("motelo")

Figura No 4. Hábitat típico de ACR Imiría, Moravec et al. '17

El estudio de Moravec (2) ubique sus puntos en el norte extremo de ACR Imiría en áreas debajo mucho presión por uso agrícola del paisaje. Mientras ninguna variación de cobertura de bosque estaba notado, este bosque se toca las orillas de la Laguna Imiría. Por eso las cifras de Moravec (2) representan un acercamiento para entender el sistema de humedales en Imiría-calificado como un sistema único en Ucayali. La diversidad en el género Osteocephalus implica la posibilidad del uso de la variedad de hábitats presentes en el sistema de este grupo muy complejo de ranas arbícolas (20, 21). 


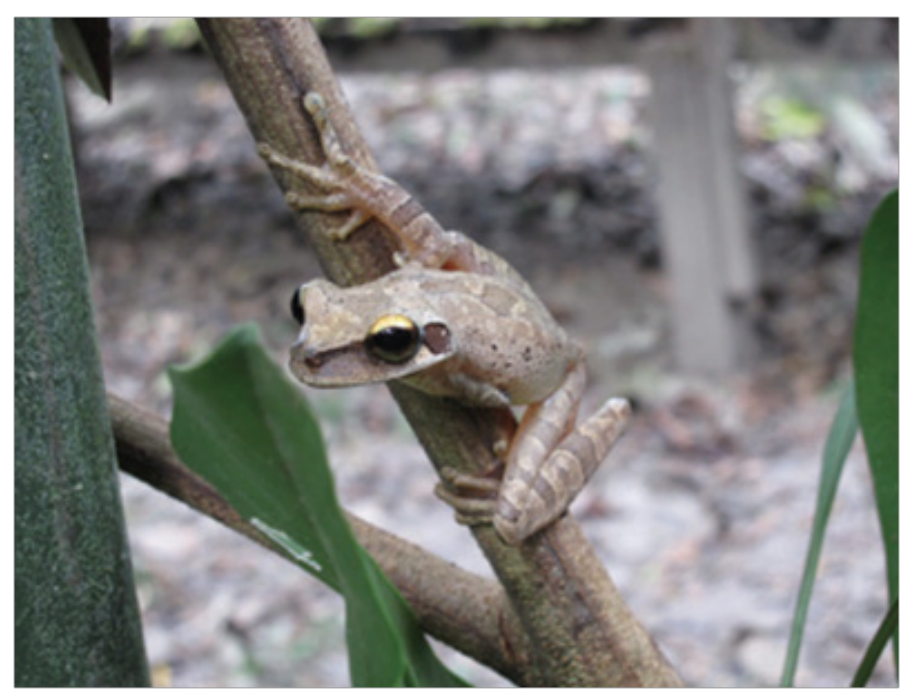

Figura No 5. Osteocephalus castaenicola - parte del complejo sin registro en Ucayali

Lista de especies:

\section{Herpetofauna (reptiles y anfibios)Área de Conservación Regional Imiría (Maisea y Nuevo Ceylán) \\ 55 especies}

\section{Ranas}

\section{Bufonidae}

La familia de los sapos

Rhinella marina

Rhinella margaritifera

Rhinella poeppigii

\section{Aromobatidae}

La familia de las ranas cohete

Allobates femoralis

\section{Dendrobatidae}

La familia de las ranas venenosas

Ameerega hahneli

\section{Craugastoridae}

La famiilia de las ranas landron de hoja rasca

Pristimantis delius

\section{Hylidae}

La familia de las ranas arbicolas

Dendropsophus parviceps

Dendropsophus leali

Dendropsophus haroldschultzi
Dendropsophus riveroi

Dendropsophus triangulum

Boana lanciformis

Boana cf. maculateralis

Boana punctata

Osteocephalus aff. yasuni

Osteocephalus planiceps

Osteocephalus taurinus

Phyllomedusa vaillanti

Phyllomedusa bicolor

Callimedusa tomopterna

PIthecopus palliata

Scinax pedromedinae

Scinax cruentommus

Scinax ruber

Scarthyla goinorum

Sphaenorynchus dorisae

Sphaenorynchus carneus

Trachycephalus typhonius

Trachycephalus cunauaru

\section{Leptodactylidae}

La familia de las ranas de lluvia

Adenomera andreae

Leptodactylus bolivianus

Leptodactylus leptodactyloides 
Leptodactylus pentadactylus ("hualo")

Lithodytes lineatus

Pipidae

Pipa pipa

Caudata

Salamandras

Bolitoglossa altamazonica

\section{Largartijas}

Dactyloidae

Anolis fuscoauratus

Anolis scypheus

\section{Teiidae}

Ameiva ameiva

\section{Hoplocercidae}

dragon de bosque

Enyaloides palpebralis

\section{Gekkota}

Gekkos

Thecadactylus solimoensis

Gonatodes humeralis

\section{Gymn-ophthalmidae}

Lagartijas enanas

Iphisa elegans

\section{Scincidae}

Copeoglossum nigropunctatum

\section{Tropiduridae}

The family of the tree runners

Uracentron flaviceps

\section{Lista completa}

La lista de especies basado en los dos estudio contiene 114 especies. Podemos aumentar la lista con algunos especies comunes notado en otras regiones por este autor, y por el gobierno regional en relación al ACR Imiría. En relación a otros regiones aliadas en Perú como Tambopata, Río Los Amigos, y la zonas del Parque Nacional Manu (22-24), esta cifra es muy bajo. Como varios sitios Amazónicos superan 200 especies, faltamos mucho effort en Ucayali para lograr una descripción completa de la diversidad herpetológica.

\section{Serpientes}

Boidae

La familia de las Boas

Epicrates cenchria

\section{Colubridae}

La familia de las serpientes inofensivas ("afaningas")

Chironius mutliventris

\section{Dipsadidae}

La familia de las culebras

Imantodes cenhcoa

Atractus aff. paravertebralis

Dipsas indica

\section{Elapidae}

La familia de las Nakka Nakka

Micrurus surinamensis

\section{Viperidae}

La familia de las viboras

Bothrops atrox ("jergon")

\section{Tortugas}

\section{Podocnemidae}

Taricaya

Podocnemis unifilis

\section{Cocodrilos}

\author{
Alligatoridae \\ La familia de los caimanes \\ Caiman crocodilus
}




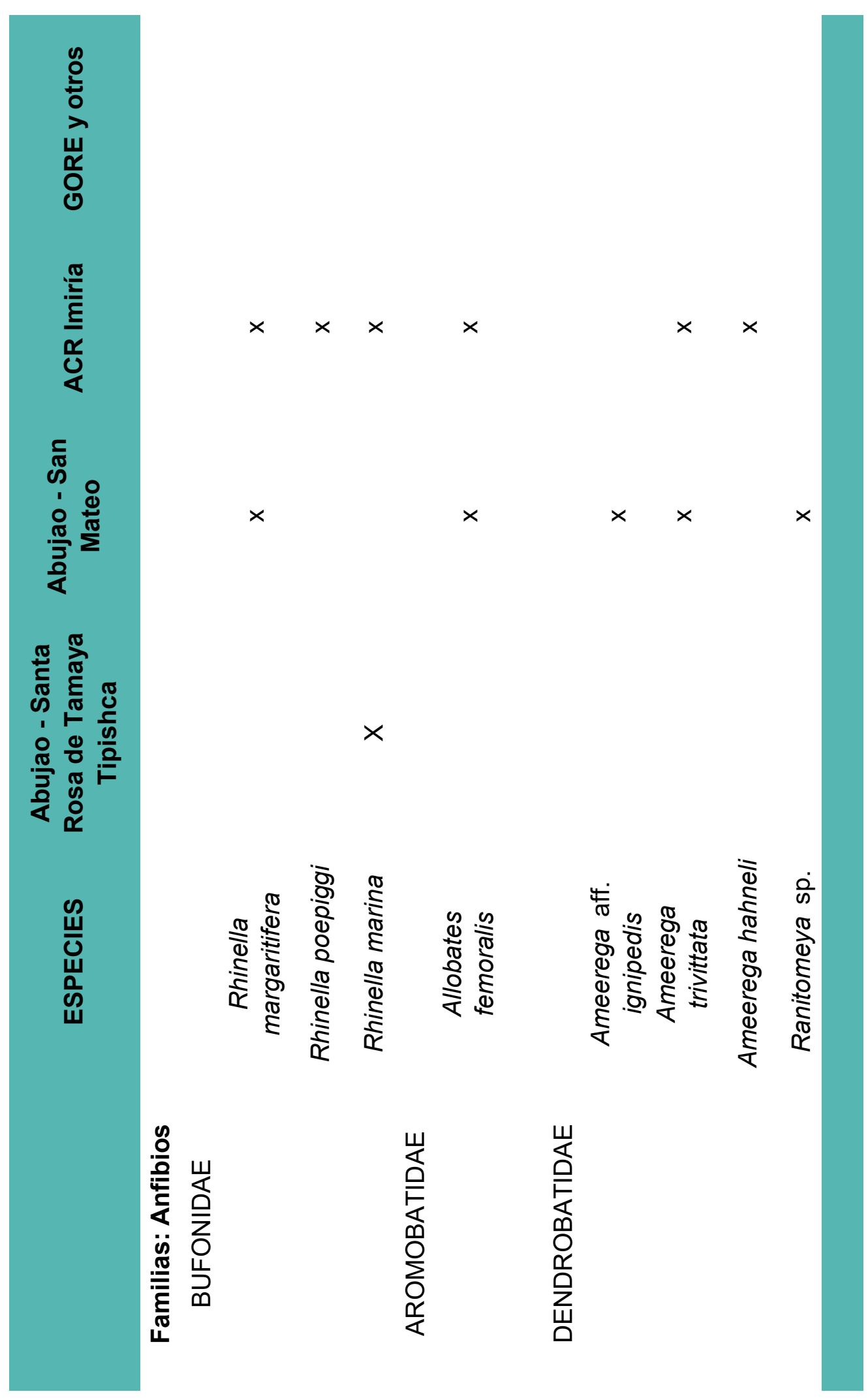




\section{Abujao - Santa \\ ESPECIES Rosa de Tamaya Tipishca Abujao - San
Mateo ACR Imiría GORE y otros}

CRAUGASTORIDAE

$\begin{array}{lc}\text { Oreobates } & \mathrm{x} \\ \text { quixensis } & \\ \text { Pristimantis } & \mathrm{x} \\ \text { achuar } & \\ \text { Pristimantis } & \mathrm{x} \\ \text { croceonguinis } & \end{array}$

$x$

$\mathrm{X}$

$\mathrm{X}$

Pristimantis delius

Pristimantis gr.

olivaceus

Pristimantis

ockendeni

Pristimantis

peruvianus

Pristimantis sp. 1

Pristimantis sp. 2

Pristimantis sp. 3

HYLIDAE

\begin{tabular}{|c|c|}
\hline \multicolumn{2}{|l|}{$\begin{array}{c}\text { Dendropsophus } \\
\text { haroldschultzi }\end{array}$} \\
\hline $\begin{array}{c}\text { Dendropsophus } \\
\text { leali }\end{array}$ & \\
\hline $\begin{array}{c}\text { Dendropsophus } \\
\text { parviceps }\end{array}$ & $x$ \\
\hline $\begin{array}{c}\text { Dendropsophus } \\
\text { cf. riveroi }\end{array}$ & \\
\hline $\begin{array}{c}\text { Dendropsophus } \\
\text { sarayacuensis }\end{array}$ & \\
\hline $\begin{array}{c}\text { Dendropsophus } \\
\text { triangulum }\end{array}$ & \\
\hline Boana boans & \\
\hline $\begin{array}{c}\text { Boana } \\
\text { geographica }\end{array}$ & $x$ \\
\hline
\end{tabular}

Boana lanciformis

Boana punctata

Boana cf. maculateralis $x$

$\mathrm{x}$

X

$x$

X

$\mathrm{X}$

$\mathrm{X}$

$\mathrm{x}$

$\mathrm{X}$

X

$\mathrm{X}$
$\mathrm{x}$
$\mathrm{x}$
$\mathrm{x}$
$\mathrm{x}$
$\mathrm{x}$
$\mathrm{x}$




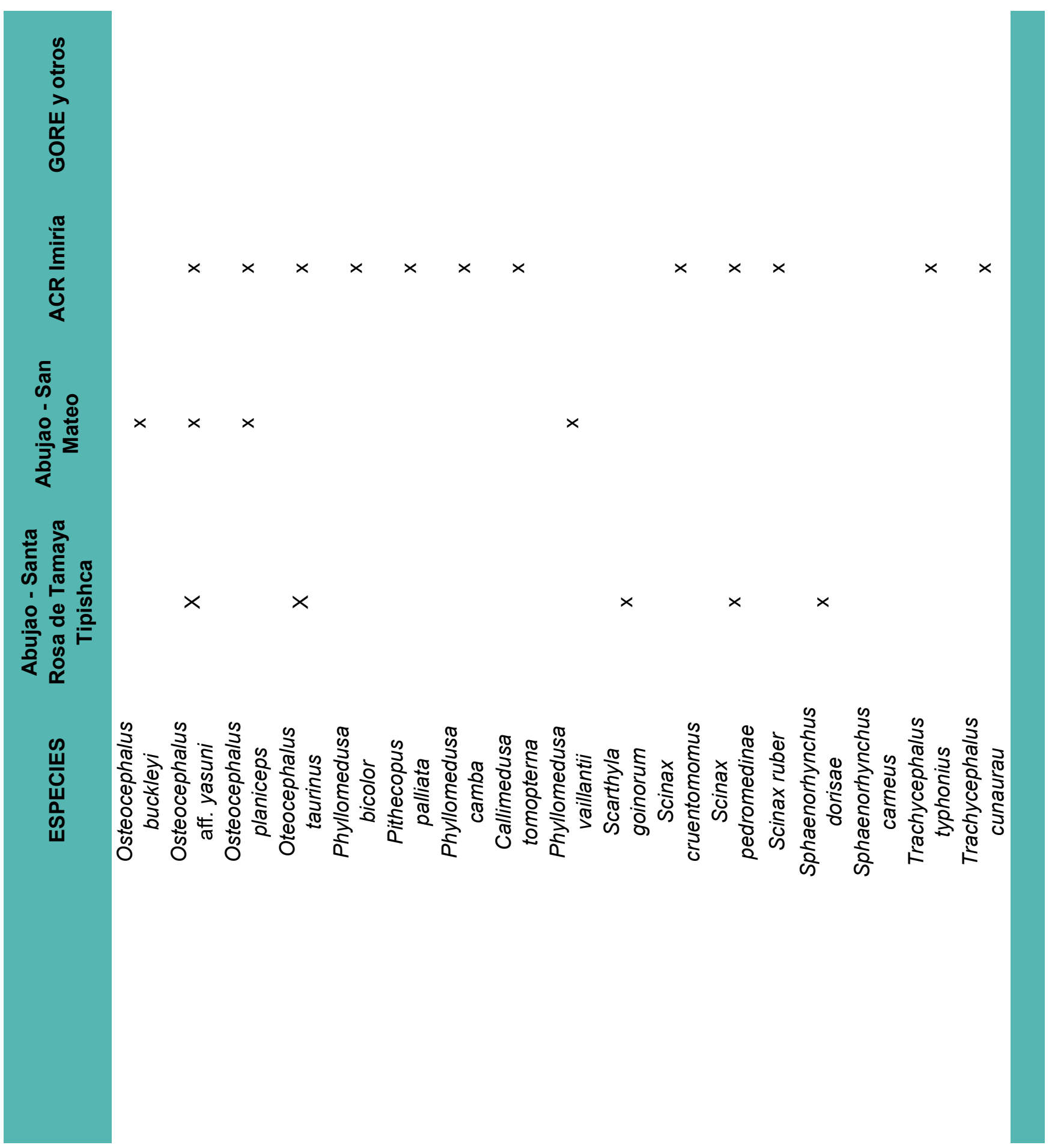




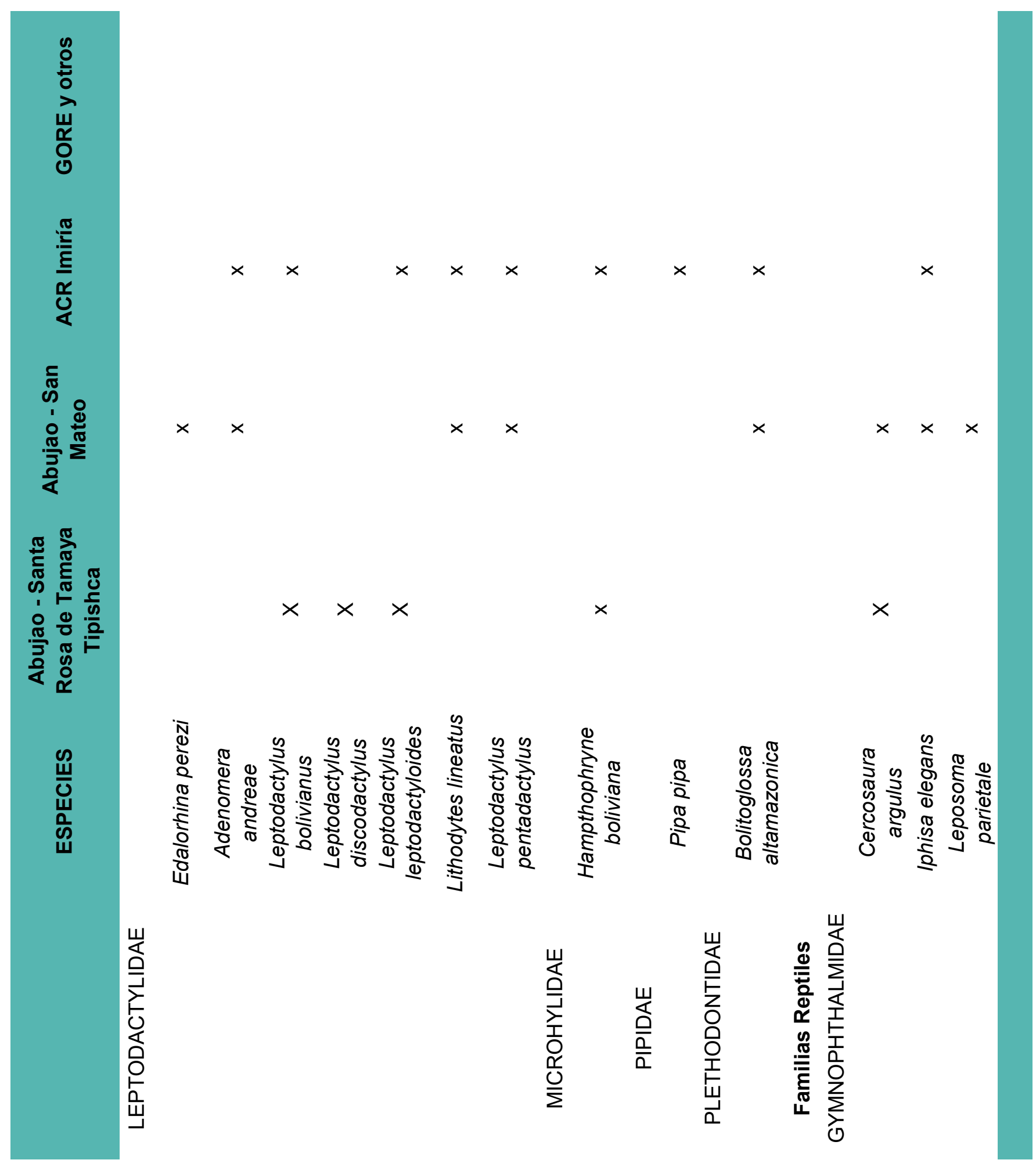




ESPECIES $\quad \begin{gathered}\text { Abujao - Santa } \\ \text { Rosa de Tamaya } \\ \text { Tipishca }\end{gathered} \quad \begin{gathered}\text { Abujao - San } \\ \text { Mateo }\end{gathered} \quad$ ACR Imiría G0RE y otros

\section{DACTYLOIDAE}

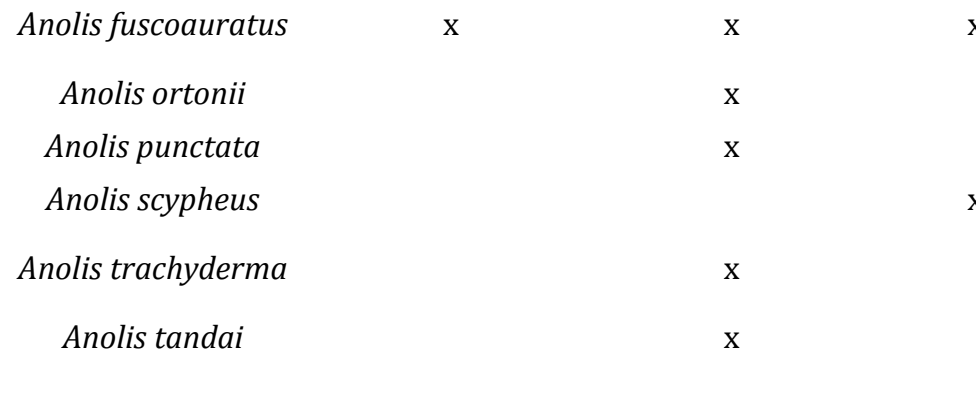

\section{Iguana iguana}

$\mathrm{X}$

$\mathrm{x}$

IGUANIDAE

SCINCIDAE

$$
\text { Copeoglossum }
$$$$
\text { nigropuntatum }
$$

orden GEKKOTA

Gonatodes
humeralis
Pseudogonatodes

TEIIDAE

$\begin{array}{ll}\text { Ameiva ameiva } & \\ \text { Dracaena } & \mathrm{X} \\ \text { guianensis } & \\ \text { Kentropyx } & \mathrm{X} \\ \text { altamazonica } & \mathrm{X} \\ \text { Kentropyx pelviceps } & \end{array}$

HOPLOCERCIDAE

TROPIDURIDAE

$$
\begin{aligned}
& \text { Enyalioides } \\
& \text { palpebralis }
\end{aligned}
$$

Plica plica

Plica umbra

Urocentron

flaviceps
$\mathrm{X}$

$\mathrm{x}$

X

$\mathrm{x}$

$\mathrm{x}$

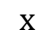

$\mathrm{x}$

$\mathrm{x}$

$\mathrm{x}$$$
\text { X }
$$ 


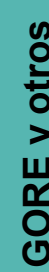

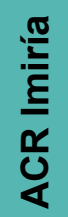

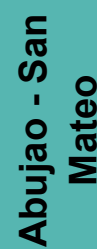

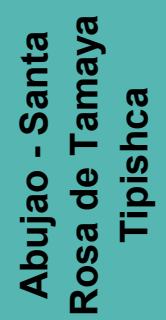

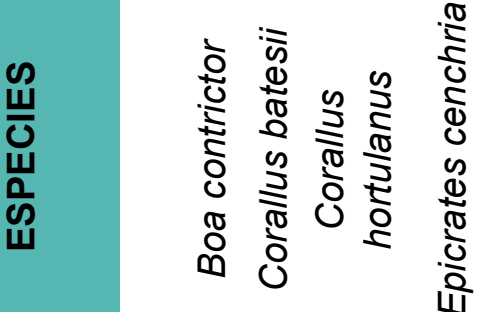

岩

$\times$

$\times$
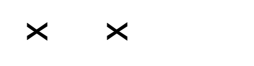
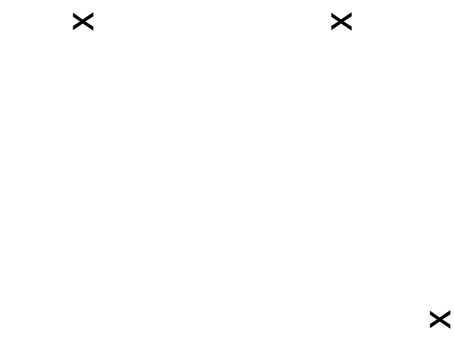

$\times$
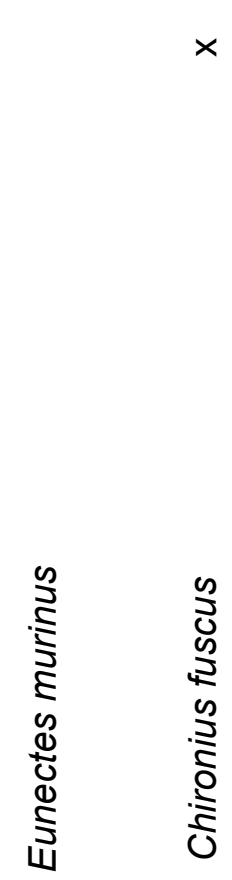

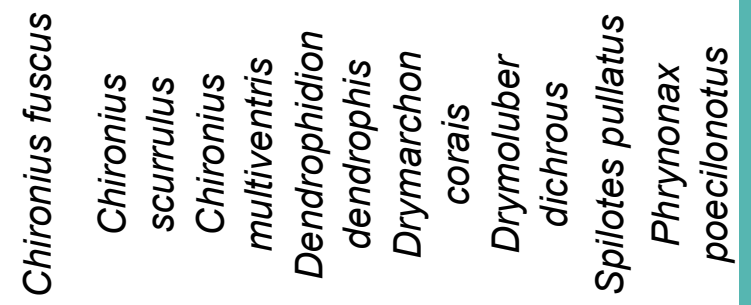

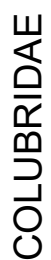




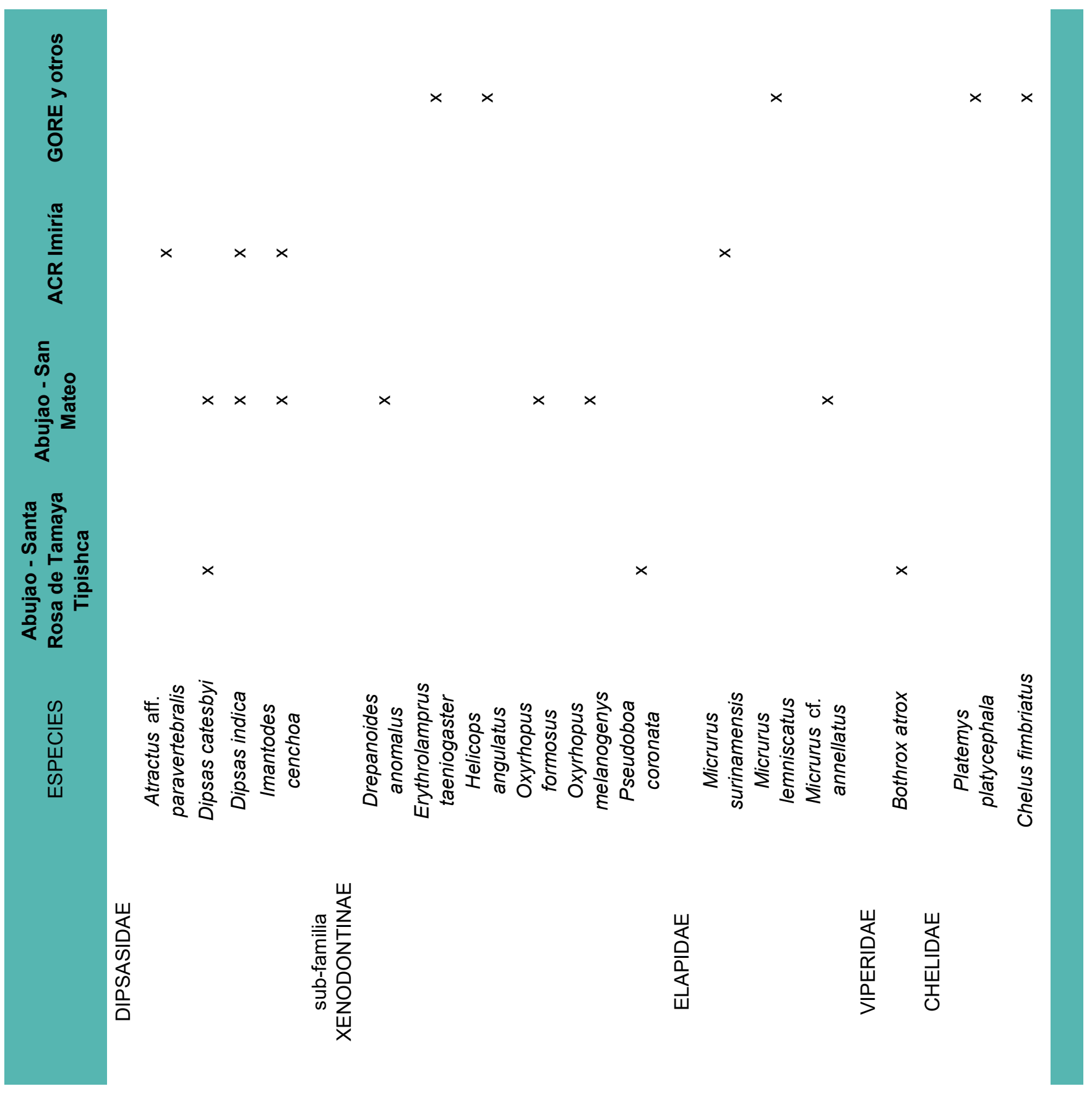




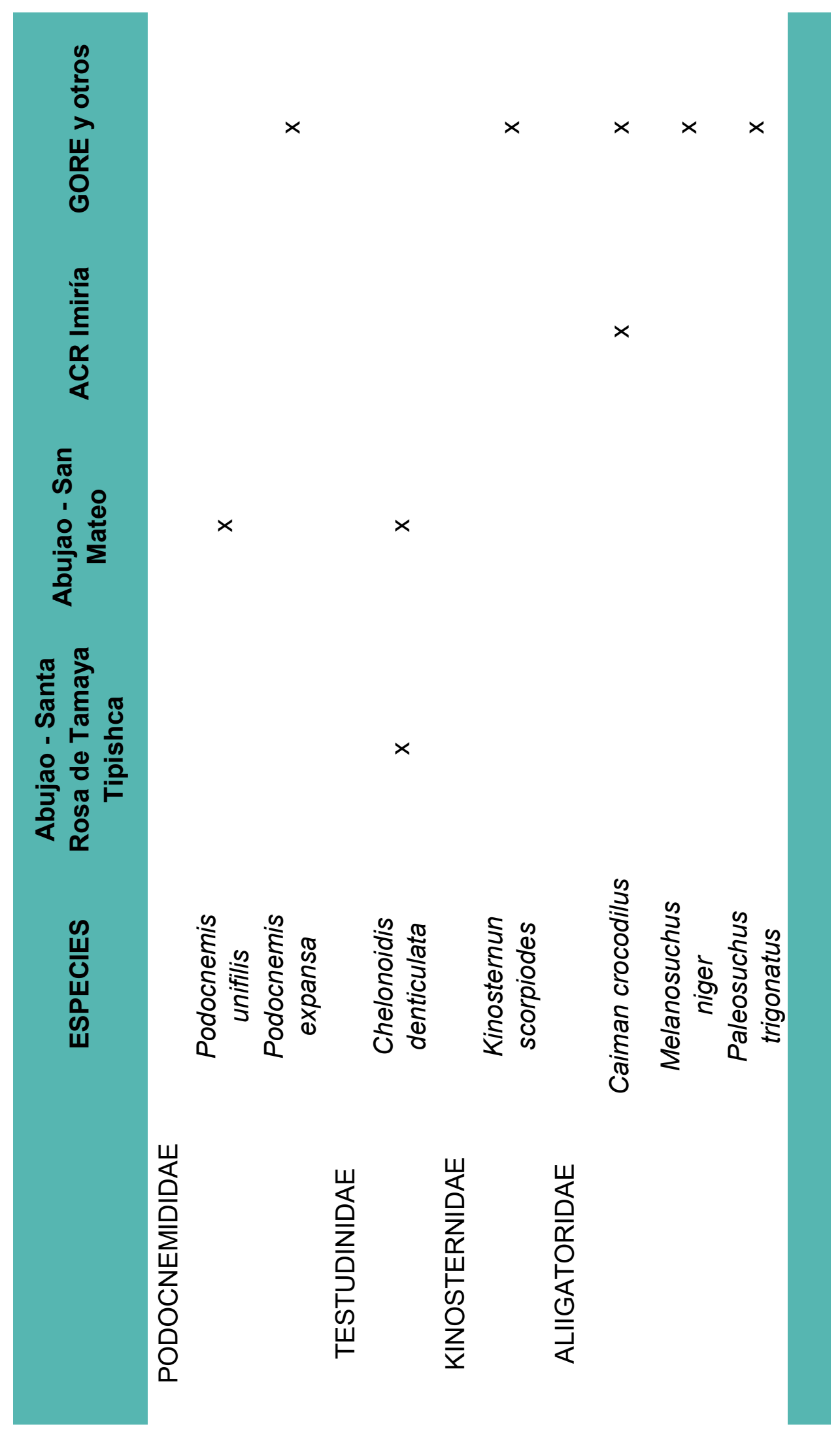




\section{CONCLUSIONES}

Hay solo dos estudios formales enfocados en anfibios y reptiles del Valle Alto-Ucayali. Uno era parte del línea base para la cuenca Abujao desarrollado por la Universidad Nacional de Ucayali. El otro es una acta del Museo Nacional en Praga, República Checa.

La variación de bosques, entre partes bajíos y el terreno firme, explica las diferencias y diversidad entre sitios. En Abujao, donde hay terrazas de terreno firme, la diversidad de ranas venenosas

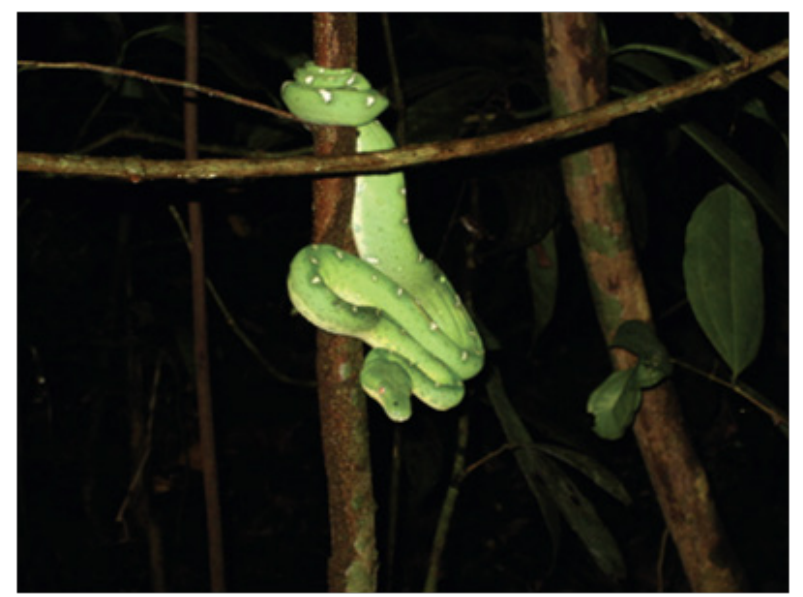

Figura № 6 . Corallus batesi $y$ Calimedusa tomopterna (familia Dendrobatidae) es más alto. El género Osteocephalus es otro indicador de diversidad de hábitats.

Las especies registradas en el Valle Alto-Ucayali llega a 114 especies, indicando que una gran diversidad falta descubrir. Como referencia, el Parque Nacional Manu tiene 240 especies, y una lista del año 1994 de la región Iquitos es 112 especies de solo anfibios (sin contar reptiles). Entonces, solo ha empezado el estudio operativo de herpetología en Ucayali.

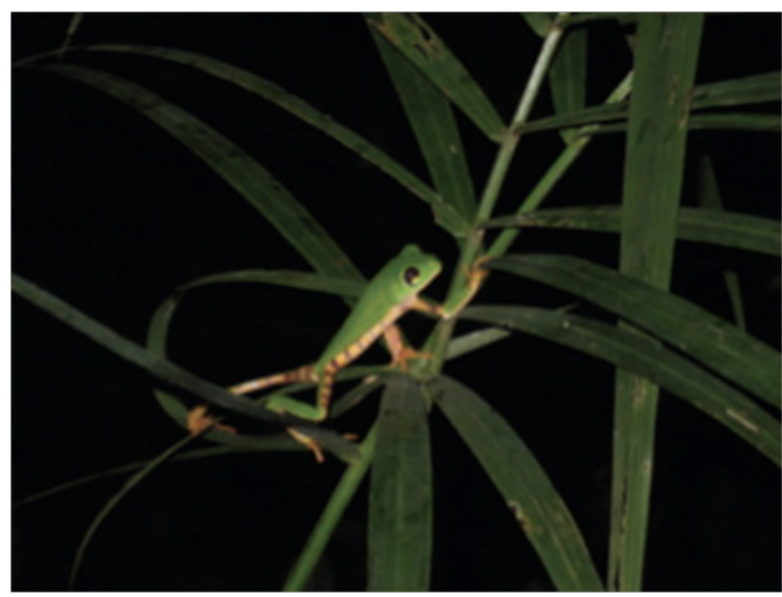

\section{BIBLIOGRÁFIA}

Gallegos Vela, Cesar Marcelo. 2015. Diseño E Implementación De Una Línea Base Ambiental Y Protocolo De Monitoreo Ambiental En La Zona De Influencia De La Zona Reservada Sierra Del Divisor Cuenca Del Río Abujao - Época Seca: Componente Herpetología. ABC LA de la Agencia de Los Estados Unidos para el Desarrollo Internacional - USAID, Universidad Nacional de Ucayali, Pucallpa.

Moravec, Jiri; Tuanama, Illich Arista; Gagliardi Urrutia, Guiseppe, Gvozdik, Vaclav. 2016. Amphibians and reptiles recorded in the Conservation Area Imiría in the Ucayali region in Peru. Acta Soc. Zool. Bohem. 80: 317-341.

Acuy Yanac, Magaly. 2014. Plan Maestro del Área de Conservación Regional Imiría. Gobierno Regional de Ucayali, Pucallpa.

J. Moravec, G. Gagliardi-Urrutia, V. Gvoždík, (2016) Amphibians and reptiles recorded in the conservation area Imi- ría in the Ucayali region in Peru, Acta Soc. Zool. Bohem. 80 317-341.

C. Vriesendorp, J. Rojas, B. Pawlak, L. Rivera (2006) Perú: Matsés, The Field Museum, Comunidad Nativa Matses, CECIA, Herbario Amazonense de la...

L.O. Rodríguez, (2003) Anfibios y reptiles de la región del Alto Purús, Alto Purús.Biodiversidad, Conservación y Manejo.Impresso Gráfica SA, Lima, Perú 89-95.

R. Von May, A. Catenazzi, A. Angulo, P.J. Venegas, C. Aguilar, (2012) Investigación y conservación de la biodiversidad en Perú: importancia del uso de técnicas modernas y procedimientos administrativos eficientes, Revista peruana de Biología. 19 351-358.

M. Goulding, Cañas C., R. Barthem, B. Forsberg, Ortega H., (2003) Amazon Headwaters: Rivers, Wildlife, and Conservation in Southeastern Peru, Asociación para la Conservación de la Cuenca Amazónica, ACCA, Lima. 
L.O. Rodríguez, W.E. Duellman, (1994) Guide to the frogs of the Iquitos region, Amazonian Peru, Natural History Museum, University of Kansas,.

W.J. Junk, M.T.F. Piedade, J. Schöngart, M. Cohn-Haft, J.M. Adeney, F. Wittmann, (2011) A classification of major naturally-occurring Amazonian lowland wetlands, Wetlands. 31 623-640.

F.C. Draper, K.H. Roucoux, I.T. Lawson, E.T. Mitchard, E.N.H. Coronado, O. Lähteenoja, L.T. Montenegro, E.V. Sandoval, R. Zaráte, T.R. Baker, (2014) The distribution and amount of carbon in the largest peatland complex in Amazonia, Environmental Research Letters. 9124017. 